\title{
Some A Posteriori Error Estimators for Elliptic Partial Differential Equations
}

\author{
By R. E. Bank and A. Weiser
}

\begin{abstract}
We present three new a posteriori error estimators in the energy norm for finite element solutions to elliptic partial differential equations. The estimators are based on solving local Neumann problems in each element. The estimators differ in how they enforce consistency of the Neumann problems. We prove that as the mesh size decreases, under suitable assumptions, two of the error estimators approach upper bounds on the norm of the true error, and all three error estimators are within multiplicative constants of the norm of the true error. We present numerical results in which one of the error estimators appears to converge to the norm of the true error.
\end{abstract}

1. Introduction. In this work, we will describe several methods for computing a posteriori error estimates for finite element calculations. That is, given some piecewise polynomial approximation $U$ to $u_{H}$, the solution of an elliptic partial differential equation, we seek some practical method for computing an estimate of $\mid\left\|u_{H}-U\right\|$ for an appropriate norm $\||\cdot|\|$. A priori estimates can give asymptotic rates of convergence as the mesh parameter $h$ tends to zero, but often cannot provide much practical information about the actual errors encountered on a given mesh with a fixed $h$. A posteriori estimates, on the other hand, attempt to provide the user of a finite element package with such information, enhancing the robustness of the package, and the reliability of the approximations it produces.

There has been a great deal of recent work by Babuška and his co-workers on local mesh refinement strategies and the a posteriori error indicators necessary for their success [4]-[10]. The indicator in [8], for example, is based on solving local Dirichlet problems in the patch of elements surrounding each vertex in the finite element mesh. In this scheme, the Dirichlet boundary conditions insure well-posedness of the local problems. Error indicators can also be based on the computation of the norm of the local residual of the elliptic equation and the jump in normal derivative of the computed solution at interelement boundaries (e.g., [9], [13]). Such schemes as a rule require less computation than the ones involving the solution of local problems. They also appear to give good results when used in local mesh refinement algorithms. However, with highly nonuniform triangular meshes, as arise with the finite element code PLTMG [11], it is sometimes difficult to weight the residual and boundary terms properly.

Received November 23, 1982; revised March 15, 1984.

1980 Mathematics Subject Classification. Primary 65N15, 65N30, 65N50.

(1985 American Mathematical Society $0025-5718 / 85 \$ 1.00+\$ .25$ per page 
The error indicators described in this paper are based on solving a local Neumann problem in each finite element. Such an approach leads directly to error indicators defined element by element, and at first seems a simple and natural approach. However, some care must be taken to insure the Neumann problems are well posed. As the element diameter tends to zero, the lower-order terms of the elliptic operator lose significance and the problems tend to singularity. To be well posed, the data (right-hand side) for these problems must be consistent in the limit $h \rightarrow 0$. The three procedures we analyze here differ in the method by which this consistency condition is satisfied. In some respects, our schemes are similar to the local residual method of Percell and Wheeler [14], but without the penalty terms.

When the approximation $U$ is the finite element approximation, our error indicators $\bar{e}=\hat{e}, \tilde{e}$, and $\check{e}$ yield error estimators $\|\hat{e}\|\|,\| \tilde{e} \|$, and $\|\check{e}\| \mid$ which satisfy the inequalities

$$
\left(1-\varepsilon_{1}\right)\left\|u_{H}-U\right\| \leqslant\|\bar{e}\| \leqslant\left(1+\varepsilon_{2}\right)\left\|u_{H}-U\right\| \|,
$$

where $\varepsilon_{1}<1$ and $\varepsilon_{2}$ is bounded. When $U$ is an arbitrary function in the finite element subspace, extra terms in (1.1) are introduced which measure the difference between $U$ and the finite element solution. Such terms are of significance if variational crimes are committed in assembling the finite element stiffness matrix and right-hand side, or if the resulting linear system is only approximately solved, say, by an iterative method.

Our theoretical results are for linear, elliptic, selfadjoint, positive-definite problems. The algorithms and many of our results extend readily to some nonselfadjoint, indefinite, and quasilinear elliptic problems. Essentially, the linear highest-order term dominates the process as the element size shrinks, so the lower-order nonlinear terms contribute only perturbations to the basic error bounds of Sections 4-6. Our results also extend with only small modifications to the situation where homogeneous Dirichlet boundary conditions are specified on all or part of $\partial \Omega$. For elements which intersect the Dirichlet boundary, a local problem with homogeneous Dirichlet boundary conditions on the Dirichlet portion of the element boundary and Neumann conditions on the remaining edges is solved. The analysis of these local problems is easier than the pure Neumann case because the consistency condition need not be satisfied.

Our error indicators are based on local computations for efficiency reasons. That is, they are calculated using computations which involve only one or a few neighboring elements at a time. Assembling a global stiffness matrix and right-hand side, and solving the resulting linear system, would generally be more expensive than computing the original solution $U$. For example, if the finite element solution $U$ is a continuous piecewise polynomial of degree $r$, a reasonable choice for an error indicator might be a discontinuous piecewise polynomial of degree $r+1$. Although the assumptions of our analysis, in particular, inequality (2.5), require the error indicators to be of higher degree than $U$, we have had practical success using continuous piecewise-linear triangular basis functions for $U$ and discontinuous piecewise-linear basis functions for the error indicators.

We can prove a local analogue of the right-hand inequality of (1.1) under some circumstances, if we replace $\left\|u_{H}-U\right\| \|_{\tau}$ with $\left\|u_{H}-U \mid\right\|_{N_{\tau}}$, where $N_{\tau}$ is a small set of elements in the neighborhood of triangle $\tau$. 
The remainder of the paper is organized as follows: Section 2 gives definitions and establishes notation. In Section 3, we give some preliminary results to be used in the analyses of our error indicators $\hat{e}, \tilde{e}$, and $\check{e}$, which are defined and analyzed in Sections 4, 5, and 6 respectively. In Section 7, we present numerical results indicating the behavior of our error indicators on several model problems.

For the error indicator $\hat{e}$ presented in Section 4 , we show that $\varepsilon_{1}$ in (1.1) tends to zero for arbitrary $U$ in the finite element space. However, we can bound $\varepsilon_{2}$ only when $u_{H}-U$ exactly satisfies a specified subset (2.6) of the orthogonalities satisfied by the finite element error, and in this case, our method for insuring a well-defined error indicator in an element requires some computations involving quantities from neighboring elements.

For the error indicator $\tilde{e}$ presented in Section 5, we can only prove that $\varepsilon_{1}$ in (1.1) tends to zero when $u_{H}-U$ satisfies the orthogonality conditions (2.6). We can always bound $\varepsilon_{2}$, with somewhat stronger bounds if $u_{H}-U$ satisfies (2.6). The computation of $\tilde{e}$ in an element requires only quantities from within that element.

For the error indicator $\check{e}$ presented in Section 6 , we bound $\varepsilon_{1}$ and $\varepsilon_{2}$. The computation of $\check{e}$ in an element requires only quantities from within that element, and requires even less work than the computation of $\tilde{e}$.

In Section 7, we find empirically that, except on very coarse grids, on several model problems our error estimators are upper bounds on the norm of the true error, and that our estimators are always within a factor of three of the norm of the true error. The simplest and cheapest of the error indicators, $\check{e}$, also performs the best for fine grids, with its norm appearing to converge to the norm of the true error as the mesh size decreases.

2. Notation. Consider the linear, selfadjoint, positive-definite Neumann problem

$$
\begin{array}{ll}
L(u)=-\nabla a \nabla u+b u=f & \text { in } \Omega \subset R^{2}, \\
a \frac{\partial u}{\partial n}=g & \text { on } \partial \Omega,
\end{array}
$$

with $\Omega$ a bounded domain, $a$ in $C^{1}(\bar{\Omega}), b$ in $C^{0}(\bar{\Omega})$. We assume there exist constants $\underline{a}, \bar{a}, \underline{b}, \bar{b}$ such that

$$
\begin{aligned}
& 0<\underline{a} \leqslant a(x) \leqslant \bar{a} \\
& 0 \leqslant \underline{b} \leqslant b(x) \leqslant \bar{b}
\end{aligned} \text { for } x \text { in } \bar{\Omega} .
$$

The boundary of $\Omega$ is assumed piecewise-smooth. The weak form of (2.1) is: find $u$ in $H^{1}(\Omega)$ such that

$$
a(u, v)=(f, v)+\langle g, v\rangle \text { for all } v \in H^{1}(\Omega),
$$

where

$$
a(u, v)=\iint_{\Omega}(a \nabla u \nabla v+b u v) d x, \quad(f, v)=\iint_{\Omega} f v d x, \quad\langle g, v\rangle=\int_{\partial \Omega} g v d s .
$$

$H^{k}(\Omega)$ for nonnegative integer $k$ will denote the usual Sobolev space equipped with the norm

$$
\|u\|_{k}^{2}=\sum_{|\beta| \leqslant k}\left\|D^{\beta} u\right\|_{0}^{2}=\sum_{|\beta| \leqslant k}\left(D^{\beta} u, D^{\beta} u\right) .
$$


Negative and nonintegral spaces will not be used. We will set $H^{1}(\Omega)=H$, and $\|\cdot\|_{0}=\|\cdot\|$. We will also use the energy norm associated with the bilinear form $a(\cdot, \cdot)$

$$
\|u\|^{2}=a(u, u) .
$$

We will consider the solution of (2.2) using a standard Rayleigh-Ritz-Galerkin procedure based on $C^{0}$ triangular finite elements. Specifically, let $F=\{T\}$ denote a family of triangulations of $\Omega$, where $T$ in $F$ is a collection of closed triangles such that, for distinct $\tau_{1}, \tau_{2}$ in $T, \tau_{1} \cap \tau_{2}$ is either empty, a single vertex, or a common edge. Triangles will normally have straight edges, although triangles with edges coincident with some part of $\partial \Omega$ will be allowed one curved boundary edge. Such an edge must be a smooth arc.

For $\tau$ in $T$, let $h_{\tau}$ denote the diameter of $\tau$ and let

$$
h=\max _{\tau \in T} h_{\tau} \text {. }
$$

Let $E$ be the collection of curves which form an edge of a triangle in $T$. The set of edges may be decomposed as the union of two disjoint sets $E=E_{B} \cup E_{I}$ where $E_{B}$ is the set of boundary edges and $E_{I}$ the set of (straight) interior edges. We denote by $E_{\tau}$ the set of three edges of the triangle $\tau$ in $T$. We define the neighbors of $\tau, N_{\tau}$, by

$$
N_{\tau}=\left\{\tau^{\prime} \in T \mid \tau^{\prime} \cap \tau \neq \varnothing\right\} .
$$

For $\varepsilon$ in $E$, let $h_{\varepsilon}$ denote the length of $\varepsilon$.

Let $\delta_{0}$ be a fixed positive constant independent of $T$ and $F$. We require each triangle $\tau$ in $T$ to be star-shaped with respect to a circle of diameter $\delta_{0} h_{\tau}$ contained in $\tau$. The constant $\delta_{0}$ is a measure of the shape regularity of the triangles in $T$. Shape regularity does not require the triangulations to be globally quasi-uniform, although it does imply a small angle condition and a local quasi-uniformity of the mesh. In particular, there exists a positive constant $\delta_{1}=\delta_{1}\left(\delta_{0}\right)$ independent of $T$ such that for $\varepsilon$ in $E_{\tau^{\prime}}, \tau^{\prime}$ in $N_{\tau}$, and $\tau$ in $T$,

$$
\delta_{1}^{-1} h_{\tau} \leqslant h_{\varepsilon} \leqslant \delta_{1} h_{\tau}
$$

For $T$ in $F$, let $\underline{S} \subseteq S \subset \bar{S}$ denote three finite-dimensional spaces of $C^{0}$ piecewise polynomials associated with $T$. The space $S$ is the space in which we will seek an approximate solution of (2.2), while $\bar{S}$ will be associated with a larger space in which we seek an approximation to the error. The space $\underline{S}$ will be a (possibly) smaller space, which contains at least the $C^{0}$ piecewise-linear functions on $T$; we allow $\underline{S}=S$.

For $Q=H, \bar{S}, S$ or $\underline{S}$, we define $u_{Q}$ in $Q$ by

$$
a\left(u_{Q}, v\right)=(f, v)+\langle g, v\rangle \quad \text { for all } v \in Q .
$$

If $b \equiv 0$, we impose the additional requirement

$$
\left(u_{Q}, 1\right)=0 \text {. }
$$

$u_{H}$ is therefore the weak solution of (2.2), while $u_{S}, u_{\bar{S}}$ and $u_{S}$ are finite element approximations of $u_{H}$. Let $U$ in $S$ denote the computed approximation of $u_{H}$ and define $e_{Q}=u_{Q}-U, Q=H, S, \bar{S}$. Normally one has $U=u_{S}$, so that $e_{S}=0$, but our analysis does not require it. Thus our error estimates will allow for the inclusion 
of effects due to roundoff errors, variational crimes, or the approximate solution of the linear equations by an iterative method.

To facilitate the introduction of the local function spaces and inner products required for our analysis, let $\omega$ be an open set in $R^{2}$ and $\gamma$ a simple piecewise-smooth curve in $R^{2}$. Let $(\cdot, \cdot)_{\omega}$ and $\|\cdot\|_{\omega}$ denote the $L^{2}(\omega)$ inner product and norm, $a(\cdot, \cdot)_{\omega}$ and $\|\mid \cdot\|_{\omega}$ denote the energy inner product and norm restricted to $\omega$, and $\langle\cdot, \cdot\rangle_{\gamma}$ and $|\cdot|_{\gamma}$ denote the inner product and norm on $L^{2}(\gamma)$.

Let $T$ in $F$ be a fixed triangulation and let

$$
H_{T}=\prod_{\tau \in T} H^{1}(\tau)=\left\{\phi|\phi|_{\tau} \in H^{1}(\tau), \tau \in T\right\}
$$

denote the space of piecewise $H^{1}$ functions. For $v, w$ in $H_{T}$ we define the broken $L^{2}$ and energy inner products and norms by

$$
\begin{aligned}
(v, w) & =\sum_{\tau \in T}(v, w)_{\tau}, & \|v\|_{0}^{2}=(v, v), \\
a(v, w) & =\sum_{\tau \in T} a(v, w)_{\tau}, & \|v\|^{2}=a(v, v) .
\end{aligned}
$$

Note that $H_{T}$ is contained in $L^{2}(\Omega)$, so the broken $L^{2}$ inner product is just the usual $L^{2}$ inner product. Also, $H(\Omega)=H^{1}(\Omega) \subset H_{T}$, and the above definitions reduce to the usual ones whenever $v, w$ are in $H(\Omega)$. Similarly, for $\Gamma=E, E_{I}, E_{B}, E_{\tau}$, or some other subset of $E$, let

$$
\langle v, w\rangle_{\Gamma}=\sum_{\varepsilon \in \Gamma}\langle v, w\rangle_{\varepsilon}, \quad|v|_{\Gamma}^{2}=\langle v, v\rangle_{\Gamma}
$$

Let $S_{\tau}$ denote the restriction of $S$ to $\tau \in T$ and let

$$
S_{T}=\prod_{\tau \in T} S_{\tau}
$$

Similarly, define $\bar{S}_{\tau}, \underline{S}_{\tau}, \underline{S}_{T}$ and $\bar{S}_{T}$. The space $S_{T}$ (and $\underline{S}_{T}, \bar{S}_{T}$ ) is a space of discontinuous piecewise polynomials locally defined in each element $\tau$ in $T$. Note the inclusions $\underline{S}_{T} \subseteq S_{T} \subset \bar{S}_{T} \subset H_{T}$.

For each edge $\varepsilon$ in $E$, we define a normal direction $n=n_{\varepsilon}$. If $\varepsilon$ is in $E_{B}$, this will be the usual outward normal. If $\varepsilon$ is in $E_{I}$, the choice is arbitrary. Since we are dealing with discontinuous spaces, it is useful to have notation describing the jump and average of functions along edges. We denote the two triangles sharing an edge $\varepsilon$ in $E_{I}$ as $\tau_{\text {in }}$ and $\tau_{\text {out }}$, where the normal $n$ is outward from $\tau_{\text {in }}$ (see Figure 2.1). Then for $x$ on $\varepsilon$,

$$
[v]_{J}(x)=\left.v(x)\right|_{\tau_{\text {sut }}}-\left.v(x)\right|_{\tau_{\text {in }}}
$$

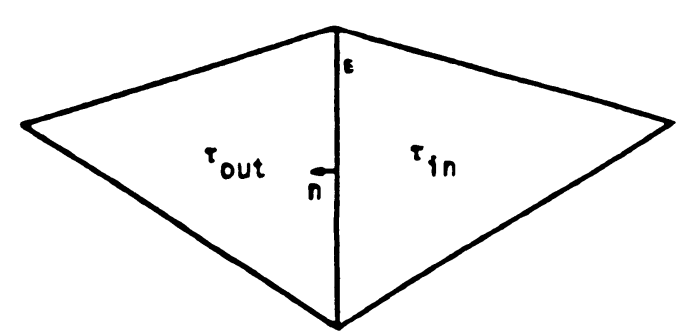

FIGURE 2.1 
is the jump in $v$ across $\varepsilon$, and

$$
[v]_{A}(x)=\frac{1}{2}\left\{\left.v(x)\right|_{\tau_{\text {out }}}+\left.v(x)\right|_{\tau_{\text {in }}}\right\}
$$

is the average of $v$ on $\varepsilon$. Note the quantity $[\partial v / \partial n]_{J}$ is independent of the direction of $n$.

Let $I: \bar{S}_{T} \rightarrow \underline{S}_{T}$ denote a local polynomial interpolation operator. For example, for each $\tau$ in $T$, we can let $I$ be the usual Lagrange interpolant for triangular elements. We assume that $I$ satisfies

(i) if $v \in \underline{S}_{T}, I v=v$ ( $I$ behaves like the identity on $\underline{S}_{T}$ : in particular, on piecewise-constant functions);

(ii) if $v \in \bar{S}, I v \in \underline{S}$ ( $I$ preserves continuity);

(iii) there exists a constant $C_{0}$, depending on $\delta_{0}, \delta_{1}, a, b$, the maximum degree of polynomials in $S$, and the particular choice of interpolation operator, but independent of $h$, such that

$$
\sup _{\substack{v \in \bar{S}_{T} \\ v \neq 0}} \frac{\|I v\|}{\|v\|} \leqslant C_{0}
$$

In Lemma 3.4 and Section 6, we will use the space

$$
\check{S}_{\tau}=\left\{v: v \text { is in } \bar{S}_{\tau} \text { and } I v=0\right\}
$$

and the product space

$$
\check{S}_{T}=\prod_{\tau \in T} \check{S}_{\tau}
$$

Note that any function $\bar{v}$ in $\bar{S}_{T}$ can be written uniquely as $\bar{v}=\underline{v}+\check{v}$, where $\underline{v}$ is in $\underline{S}_{T}$ and $\check{v}$ is in $\check{S}_{T}$.

Let $\tau$ be in $T$ and $\varepsilon$ be in $E_{\tau}$. In our analyses, we will use the following inequalities

$$
\begin{gathered}
|v|_{E_{\tau}}^{2} \leqslant C_{1}^{2}\left\{h_{\tau}^{-1}\|v\|_{\tau}^{2}+h_{\tau}\|\nabla v\|_{\tau}^{2}\right\}, \quad v \in H^{1}(\tau), \\
\left|\frac{\partial v}{\partial n}\right|_{E_{\tau}}^{2} \leqslant C_{1}^{2}\left\{h_{\tau}^{-1}\|\nabla v\|_{\tau}^{2}+h_{\tau}\left\|\nabla^{2} v\right\|_{\tau}^{2}\right\}, \quad v \in H^{2}(\tau), \\
\left\|\nabla^{p}\right\|_{\tau} \leqslant C_{2} h_{\tau}^{q-p}\left\|\nabla^{q} v\right\|_{\tau}, \quad v \in \bar{S}, 0 \leqslant q \leqslant p \leqslant 2 .
\end{gathered}
$$

The trace inequalities $(2.4 \mathrm{a}-\mathrm{b})$ follow from results in Agmon [1]; the constant $C_{1}=C_{1}\left(\delta_{0}, \delta_{1}\right)$. Inequality $(2.4 \mathrm{c})$ is a local inverse inequality; the constant $C_{2}$ depends on $\delta_{0}$ and the maximum degree of polynomials contained in $\bar{S}_{T}$. Both $C_{1}$ and $C_{2}$ are independent of $h$.

We require some notion of convergence of the finite element solutions $u_{S}$ and $u_{\bar{S}}$ to the weak solution $u_{H}$ of (2.2) as a function of $h$. In particular, we make the saturation assumption

$$
\left\|u_{H}-u_{\bar{S}}\right\|^{2}+\left|h_{\varepsilon}^{1 / 2}\left[a \frac{\partial\left(u_{H}-u_{\bar{S}}\right)}{\partial n}\right]_{A}\right|_{E_{I}}^{2} \leqslant \beta^{2}\left\|u_{H}-u_{S}\right\|^{2},
$$

where $\beta=\beta(h)$ and $\lim _{h \rightarrow 0} \beta=0$ (Babuška [2], Babuška and Luskin [3]). This assumption is reasonable, since $\bar{S}$ contains piecewise polynomials of higher degree than $S$. 
Finally, some of our results will hold only when $U$ satisfies

$$
a\left(e_{S}, x\right)=a\left(u_{S}-U, x\right)=0 \text { for all } x \in \underline{S},
$$

i.e., the elliptic projection of the error on $\underline{S}$ is zero. Note that (2.6) holds if $U$ is the finite element solution $u_{S}$ or if $U=u_{\underline{s}}$. It may also hold when $U$ is computed using certain two-level iterative schemes, where the coarse level corresponds to $\underline{S}$. If the iteration terminates on a coarse subspace correction, then the approximate solution $U$ will automatically satisfy (2.6).

3. Preliminary Results. In this section we present some results which are useful in analyzing the error estimation procedures to be discussed in Sections 4-6. Let $e_{Q}=u_{Q}-U$ for $Q=H, \underline{S}, S, \bar{S}$. Then for $v$ in $Q$, using (2.3a) and integration by parts in each element,

$$
\begin{aligned}
a\left(e_{Q}, v\right) & =(f, v)+\langle g, v\rangle-\sum_{\tau \in T}\left\langle a \frac{\partial U}{\partial n_{\tau}}, v\right\rangle_{E_{\tau}}-(L(U), v) \\
& =(r, v)+\left\langle r_{B}, v\right\rangle-\sum_{\tau \in T}\left\langle a \frac{\partial U}{\partial n_{\tau}}, v\right\rangle_{E_{\tau} \cap E_{I}} .
\end{aligned}
$$

Here $n_{\tau}$ is the outward normal for $\tau$. $r=f-L(U)$ is defined elementwise with possible discontinuities on $E_{I} \cdot r_{B}=g-a \partial U / \partial n$ is defined edgewise on $E_{B}$.

Noting the outward normal for $\tau$ is the inward normal for a neighbor sharing a common edge, we may write the last term in (3.1) as

$$
-\sum_{\tau \in T}\left\langle a \frac{\partial U}{\partial n_{\tau}}, v\right\rangle_{E_{\tau} \cap E_{I}}=\left\langle\left[a \frac{\partial U}{\partial n}\right]_{J}, v\right\rangle_{E_{I}} .
$$

We define the local right-hand side of (3.1) by

$$
F_{\tau}(v)=(r, v)_{\tau}+\left\langle r_{B}, v\right\rangle_{E_{\tau} \cap E_{B}}+\frac{1}{2}\left\langle\left[a \frac{\partial U}{\partial n}\right]_{J}, v\right\rangle_{E_{\tau} \cap E_{I}}
$$

for $v$ in $Q_{\tau}$. Summing over triangles, we obtain the linear functional $F(v)$, where

$$
F(v)=\sum_{\tau \in T} F_{\tau}(v)=(r, v)+\left\langle r_{B}, v\right\rangle+\left\langle\left[a \frac{\partial U}{\partial n}\right]_{J},[v]_{A}\right\rangle_{E_{I}}
$$

for $v$ in $Q_{T}$.

In terms of this linear functional, (3.1) can be written as

$$
a\left(e_{Q}, v\right)=F(v) \text { for all } v \in Q
$$

Equation (3.4) gives a useful characterization of the error. Noting $e_{H}-e_{Q}=u_{H}-u_{Q}$ $(Q=\underline{S}, S, \bar{S}),(3.4)$ implies the orthogonality relations

$$
a\left(e_{H}-e_{Q}, v\right)=0, \quad v \in Q=\underline{S}, S, \bar{S},
$$

normally associated with the error in the finite element method, as well as

$$
\begin{array}{ll}
a\left(e_{\bar{S}}-e_{Q}, v\right)=0, & v \in Q=\underline{S}, S, \\
a\left(e_{S}-e_{\underline{S}}, v\right)=0, & v \in \underline{S} .
\end{array}
$$

The orthogonality relations (3.5) imply

$$
\begin{array}{ll}
\left\|e_{H}\right\|\left\|^{2}=\right\| e_{Q}\left\|^{2}+\right\| e_{H}-e_{Q} \|^{2}, & Q=\underline{S}, S, \bar{S} ; \\
\left\|e_{\bar{S}}\right\|^{2}=\left\|e_{Q}\right\|^{2}+\left\|e_{\bar{S}}-e_{Q}\right\|^{2}, & Q=\underline{S}, S ; \\
\left\|e_{S}\right\|^{2}=\left\|e_{\underline{S}}\right\|^{2}+\left\|e_{S}-e_{\underline{S}}\right\|^{2} . &
\end{array}
$$


Equation (3.4) characterizes the inner product $a\left(e_{Q}, v\right)$ when $v$ is in $Q$. For $e_{H}$, we will also need to characterize the inner product $a\left(e_{H}, v\right)$ for $v$ in $H_{T}$. To do so, we start from the elementwise version of (3.1). Suppose $e_{H}$ is in $H^{2}(\tau)$; then, integrating by parts,

$$
a\left(e_{H}, v\right)_{\tau}=(r, v)_{\tau}+\left\langle r_{B}, v\right\rangle_{E_{\tau} \cap E_{B}}+\left\langle a \frac{\partial e_{H}}{\partial n_{\tau}}, v\right\rangle_{E_{\tau} \cap E_{I}}
$$

for all $v$ in $H^{1}(\tau)$. In summing over elements, the third term is somewhat more complicated than in $(3.1)$, since $[v]_{J} \neq 0$. In particular,

$$
\sum_{\tau \in T}\left\langle a \frac{\partial e_{H}}{\partial n_{\tau}}, v\right\rangle_{E_{\tau} \cap E_{I}}=\left\langle\left[a \frac{\partial U}{\partial n}\right]_{J},[v]_{A}\right\rangle_{E_{I}}+\left\langle\left[a \frac{\partial e_{H}}{\partial n}\right]_{A},[v]_{J}\right\rangle_{E_{I}} .
$$

This gives the global equation

$$
a\left(e_{H}, v\right)=F(v)+\left\langle\left[a \frac{\partial e_{H}}{\partial n}\right]_{A},[v]_{J}\right\rangle_{E_{I}} \quad \text { for all } v \in H_{T} .
$$

Note that (3.8) reduces to (3.4) whenever $v$ is in $H$.

In the course of our analysis in Sections 4-6, there are several basic estimates that will be used often. We summarize these in Lemmas 3.1-3.3.

Lemma 3.1. Assume (2.4b-c) and (2.5) hold. Then

$$
\mid h_{\varepsilon}^{1 / 2}\left[a \frac{\partial e_{H}}{\partial n}\right]_{A} \leqslant C_{E_{l}}\left\|e_{H}\right\|
$$

where $C_{3}=C_{3}\left(\underline{a}, \bar{a}, C_{1}, C_{2}, \delta_{1}, \beta\right)$.

Proof. First, note

$$
\left|h_{\varepsilon}^{1 / 2}\left[a \frac{\partial e_{H}}{\partial n}\right]_{A}\right|_{E_{I}} \leqslant\left|h_{\varepsilon}^{1 / 2}\left[a \frac{\partial e_{\bar{S}}}{\partial n}\right]_{A}\right|_{E_{I}}+\left|h_{\varepsilon}^{1 / 2}\left[a \frac{\partial\left(u_{H}-u_{\bar{S}}\right)}{\partial n}\right]_{A}\right|_{E_{I}} .
$$

The second term can be bounded using (2.5),

$$
\left|h_{\varepsilon}^{1 / 2}\left[a \frac{\partial\left(u_{H}-u_{\bar{S}}\right)}{\partial n}\right]_{A}\right|_{E_{I}} \leqslant \beta\left\|u_{H}-u_{\bar{S}}\right\| \leqslant \beta\left\|e_{H}\right\| .
$$

To bound the first term, let $\varepsilon$ be in $E_{I}$ and $\tau_{\text {in }} \cap \tau_{\text {out }}=\varepsilon$. Then

$$
\left|h_{\varepsilon}^{1 / 2}\left[a \frac{\partial e_{\bar{S}}}{\partial n}\right]_{A}\right|_{\varepsilon}^{2} \leqslant \frac{h_{\varepsilon}}{2}\left\{\left.\left|a \frac{\partial e_{\bar{S}}}{\partial n}\right|_{\tau_{\text {in }}}\right|_{\varepsilon} ^{2}+\left.\left|a \frac{\partial e_{\bar{S}}}{\partial n}\right|_{\tau_{\text {out }}}\right|_{\varepsilon} ^{2}\right\} \text {. }
$$

Using $(2.4 b-c)$ we obtain

$$
\begin{aligned}
\left.h_{\varepsilon}\left|a \frac{\partial e_{\bar{S}}}{\partial n}\right|_{\tau}\right|_{\varepsilon} ^{2} & \leqslant \bar{a}^{2} C_{1}^{2} h_{\varepsilon}\left\{h_{\tau}^{-1}\left\|\nabla e_{\bar{S}}\right\|_{\tau}^{2}+h_{\tau}\left\|\nabla^{2} e_{\bar{S}}\right\|_{\tau}^{2}\right\} \\
& \leqslant \bar{a}^{2} C_{1}^{2} \delta_{1}\left(1+C_{2}^{2}\right)\left\|\nabla e_{S}\right\|^{2} \\
& \leqslant \bar{a}^{2} \underline{a}^{-1} C_{1}^{2} \delta_{1}\left(1+C_{2}^{2}\right)\left\|e_{S}\right\|_{\tau}^{2} .
\end{aligned}
$$

Summing over edges, and using the fact that each triangle has at most 3 edges, we obtain

$$
\left|h_{\varepsilon}^{1 / 2}\left[a \frac{\partial e_{\bar{S}}}{\partial n}\right]_{A}\right|_{E_{I}}^{2} \leqslant \frac{3}{2} \bar{a}^{2} \underline{a}^{-1} C_{1}^{2} \delta_{1}\left(1+C_{2}^{2}\right)\left\|e_{S}\right\|^{2} .
$$

Combining (3.9), (3.10) and (3.6) proves the lemma. 
LEMMA 3.2. Let $v$ be in $H_{T}$, and suppose

$$
\|v\|_{\tau} \leqslant C_{4} h_{\tau}\|v\|_{\tau} \text { for all } \tau \in T,
$$

where $C_{4}$ is independent of $\tau$ and $v$. Then,

$$
\left|h_{\varepsilon}^{-1 / 2}[v]_{J}\right|_{E_{I}} \leqslant C_{5}\|v\| \|
$$

where $C_{5}=C_{5}\left(C_{1}, C_{4}, \underline{a}, \delta_{1}\right)$.

Proof. As in the proof of Lemma 3.1,

$$
\left|[v]_{J}\right|_{\varepsilon}^{2} \leqslant 2\left(\left.|v|_{\tau_{\text {in }}}\right|_{\varepsilon} ^{2}+\left.|v|_{\tau_{\text {out }}}\right|_{\varepsilon} ^{2}\right)
$$

Using (2.4a) and the hypothesis of the lemma, we obtain

$$
\left.|v|_{\tau}\right|_{\varepsilon} ^{2} \leqslant C_{1}^{2}\left(h_{\tau}^{-1}\|v\|_{\tau}^{2}+h_{\tau}\|\nabla v\|_{\tau}^{2}\right) \leqslant C_{1}^{2}\left(1+C_{4}^{2}\right) \underline{a}^{-1} \delta_{1} h_{\varepsilon}\|v\|_{\tau}^{2} .
$$

Thus, summing over the edges,

$$
\left|h_{\varepsilon}^{-1 / 2}[v]_{J}\right|_{E_{l}}^{2} \leqslant 6 C_{1}^{2}\left(1+C_{4}^{2}\right) \underline{a}^{-1} \delta_{1}\|v\| \|^{2}
$$

and the lemma follows.

Lemma 3.3. Assume (2.5) holds and $\beta<1$. Then,

$$
\left(1-\beta^{2}\right)^{1 / 2}\left\|e_{H}\right\| \leqslant\left\|e_{\bar{s}}\right\| .
$$

Proof. Using (3.6a) for the cases $Q=\bar{S}$ and $Q=S$, and (2.5), we have

$$
\left\|e_{H}\right\|^{2} \leqslant \beta^{2}\left\|e_{H}-e_{S}\right\|^{2}+\left\|e_{\bar{S}}\right\|^{2} \leqslant \beta^{2}\left\|e_{H}\right\|^{2}+\left\|e_{\bar{S}}\right\|^{2}
$$

and the result follows.

In Section 6, we will use the following strengthened Cauchy-Schwarz inequality, proved in Bank and Dupont [12].

LEMMA 3.4. There exists a constant $\gamma<1$ depending on $\delta_{0}, \delta_{1}, a, b$, the maximum degreee of polynomials in $\bar{S}$, and the particular choice of interpolation operator $I$, but independent of $h$, such that

$$
|a(\underline{v}, \check{v})| \leqslant \gamma\|\underline{v}|\||\|\check{v}\||
$$

for any $\underline{v}$ in $\underline{S}_{T}$ and $\check{v}$ in $\check{S}_{T}$.

4. An Error Estimator. Let $\theta$ in $L^{2}(E)$ be a function defined on each edge $\varepsilon$, with $\left.\theta\right|_{E_{B}} \equiv 0$. If $\varepsilon=\bar{\tau}_{\text {in }} \cap \bar{\tau}_{\text {out }}$, define

$$
\theta_{\tau_{\text {out }}}=\theta, \quad \theta_{\tau_{\text {in }}}=-\theta
$$

so that

$$
\sum_{\tau \in T}\left\langle\theta_{\tau}, v\right\rangle_{E_{\tau}}=\left\langle\theta,[v]_{J}\right\rangle_{E_{I}}
$$

for all $v \in \bar{S}_{T}$.

Given $\theta$, we can formulate equations in each $\tau$ for the error indicator $\hat{e}$ in $\bar{S}_{T}$ as follows:

$$
a(\hat{e}, v)_{\tau}=F_{\tau}(v)+\left\langle\theta_{\tau}, v\right\rangle_{E_{\tau} \cap E_{I}}
$$


for all $v$ in $\bar{S}_{\tau}$. For the moment, assume $\theta$ is chosen so that (4.1) makes $\hat{e}$ well-defined. Appropriate choices for $\theta$ will be discussed shortly. Summing over triangles, $\hat{e}$ satisfies the global equations

$$
a(\hat{e}, v)=F(v)+\left\langle\theta,[v]_{J}\right\rangle_{E_{I}}
$$

for all $v$ in $\bar{S}_{T}$.

We immediately have the following:

THEOREM 4.1. If ê exists, $\left(1-\beta^{2}\right)^{1 / 2}\|\| e_{H}\|\leqslant\| \hat{e} \|$.

Proof. Since $e_{\bar{S}}$ is in $\bar{S},\left[e_{\bar{S}}\right]_{J} \equiv 0$, and by (3.4) and (4.2),

$$
a\left(e_{\bar{S}}, e_{\bar{S}}\right)=F\left(e_{\bar{S}}\right)=a\left(\hat{e}, e_{\bar{S}}\right)
$$

so $\left\|e_{\bar{s}}\right\| \leqslant\|\hat{e}\|$. The theorem now follows from Lemma 3.3.

There are several ways to choose $\theta$ such that $\hat{e}$ is well-defined.

If $\underline{b}>0$, we can simply choose $\theta \equiv 0$. Unfortunately, this may allow $\|\hat{e}\|$ to be much larger than $\left\|\mid e_{H}\right\|$. Another possibility is to let $\theta$ be an approximation to $\left[a \partial e_{H} / \partial N\right]_{A}$ (see Eq. (4.8)).

If $b \equiv 0$, since $a(v, 1)_{\tau}=0$ for any $v$ in $\bar{S}_{\tau}$, we will require the $\theta_{\tau}$ to satisfy

$$
\left\langle\theta_{\tau}, 1\right\rangle_{E_{\tau}}=-F_{\tau}(1) \text {. }
$$

Then (4.1) will be consistent and $\hat{e}$ will be well-defined. Summing over any arbitrary set of triangles $T^{0},(4.3)$ implies

$$
\sum_{\tau \in T^{0}}\left\langle\theta_{\tau}, 1\right\rangle_{E_{\tau}}=-\sum_{\tau \in T^{0}} F_{\tau}(1) \text {. }
$$

By (2.3b) and (3.4), $F(1)=0$, so (4.4) holds for $T^{0}=T$. Now suppose $T^{1}=T^{2} \cup T^{3}$ is a set of triangles, $T^{2} \cap T^{3}=\varnothing$, and we have chosen $\theta$ on $\partial T^{1}$ such that (4.4) holds for $T^{0}=T^{1}$. Then on $\partial T^{2}-\partial T^{1}$, we can choose $\theta$ such that (4.4) holds for $T^{0}=T^{2}$. For example, we can set $\theta$ to be the constant

$$
\left.\theta_{\tau}\right|_{\partial T^{2}-\partial T^{1}}=\frac{\sum_{\tau \in T^{2}} F_{\tau}(1)-\left\langle\theta_{\tau}, 1\right\rangle_{\partial T^{2} \cap \partial T^{1}}}{\sum_{\varepsilon \in \partial T^{2}-\partial T^{1}} h_{\varepsilon}} .
$$

The best choice of $\left.\theta_{\tau}\right|_{\partial T^{2}-\partial T^{1}}$ is an open question. Then (4.4) automatically holds for $T^{0}=T^{3}$. Continuing in this manner insures (4.4) holds for all triangles; always choosing $T^{2}$ to be a single triangle allows local computation of $\theta$. Unfortunately, this manner of choosing $\theta$ may again allow $\|\hat{e}\| \mid$ to be much larger than $\left\|e_{H}\right\|$.

We now show a way of choosing $\theta$ when (2.6) holds, which insures both that $\hat{e}$ is well-defined, and that $\|\hat{e} \mid\|$ is not too much bigger than $\left\|\mid e_{H}\right\| \|$.

Let $\left\{x_{i}\right\}$ be the vertices of the triangulation $T$, and let $\left\{b_{i}\right\}$ be the usual Lagrange basis for the $C^{0}$ piecewise linear functions on $T$, with $b_{i}\left(x_{j}\right)=\delta_{i j}$.

Recall $\underline{S}$ contains $\left\{b_{i}\right\}$. We will choose $\theta$ to be a linear function on each edge $\varepsilon$, such that

$$
\left\langle\theta_{\tau}, b_{i}\right\rangle_{E_{\tau}}=-F_{\tau}\left(b_{i}\right)
$$

for all $b_{i}$ and $\tau$. In particular, since $\sum_{i} b_{i} \equiv 1,(4.3)$ will hold, and $\hat{e}$ will always be well-defined.

Let $\nu(\varepsilon, j)$ be an indexing function such that edge $\varepsilon$ connects vertices $x_{\nu(\varepsilon, 0)}$ and $x_{\nu(\varepsilon, 1)}$, and define

$$
\sigma_{\varepsilon, j}(x)=\frac{4 b_{\nu(\varepsilon, j)}(x)-2 b_{\nu(\varepsilon, 1-j)}(x)}{h_{\varepsilon}}, \quad j=0,1,
$$


for $x$ in $\varepsilon$. By construction,

$$
\left\langle\sigma_{\varepsilon, j}, b_{\nu(\varepsilon, k)}\right\rangle_{\varepsilon}=\delta_{j k}, \quad j, k=0,1 .
$$

Expressing $\theta$ as a linear combination of the $\sigma_{\varepsilon, j}$ 's,

$$
\boldsymbol{\theta}=\sum_{\varepsilon \in E} \sum_{j=0,1} \boldsymbol{\theta}_{\varepsilon, j} \sigma_{\varepsilon, j} .
$$

Eqs. (4.5) decouple into sets of equations for each $b_{i}$,

$$
\sum_{\nu(\varepsilon, j)=i} R_{\tau, \varepsilon} \theta_{\varepsilon, j}=-F_{\tau}\left(b_{i}\right)
$$

for all $\tau$ in $\operatorname{supp}\left(b_{i}\right)$, where

$$
R_{\tau, \varepsilon}=\left\{\begin{aligned}
1 & \text { if } \tau=\tau_{\text {out }}(\varepsilon), \\
-1 & \text { if } \tau=\tau_{\text {in }}(\varepsilon)
\end{aligned}\right.
$$

For example, for the vertex $x_{i}$ depicted in Figure 4.1, the resulting system of linear equations is

$$
\left[\begin{array}{rrrr}
1 & 0 & 0 & -1 \\
-1 & 1 & 0 & 0 \\
0 & -1 & 1 & 0 \\
0 & 0 & -1 & 1
\end{array}\right]\left[\begin{array}{l}
\theta_{\varepsilon_{0}, j_{0}} \\
\boldsymbol{\theta}_{\varepsilon_{1}, j_{1}} \\
\boldsymbol{\theta}_{\varepsilon_{2}, j_{2}} \\
\boldsymbol{\theta}_{\varepsilon_{3}, j_{3}}
\end{array}\right]=\left[\begin{array}{l}
-F_{\tau_{0}}\left(b_{i}\right) \\
-F_{\tau_{1}}\left(b_{i}\right) \\
-F_{\tau_{2}}\left(b_{i}\right) \\
-F_{\tau_{3}}\left(b_{i}\right)
\end{array}\right],
$$

where $i=\nu\left(\varepsilon_{0}, j_{0}\right)=\nu\left(\varepsilon_{1}, j_{1}\right)=\nu\left(\varepsilon_{2}, j_{2}\right)=\nu\left(\varepsilon_{3}, j_{3}\right)$. The matrix has exactly one eigenvector $[1,1,1,1]^{T}$ with eigenvalue zero. However, since $b_{i}$ is in $\underline{S}, a\left(e_{H}, b_{i}\right)=$ $F\left(b_{i}\right)=0$, and thus

$$
-\sum_{k=0}^{3} F_{\tau_{k}}\left(b_{i}\right)=0,
$$

so the system is consistent. One solution of the system is

$$
\boldsymbol{\theta}_{\varepsilon_{m}, j_{m}}=-\sum_{k=0}^{m} F_{\tau_{k}}\left(b_{i}\right),
$$

so that we can choose $\theta$ such that

$$
\left|\theta_{\varepsilon, j}\right| \leqslant \sum_{\tau_{k} \in \operatorname{supp}\left(b_{i}\right)}\left|F_{\tau_{k}}\left(b_{i}\right)\right|
$$

where $i=\nu(\varepsilon, j)$.

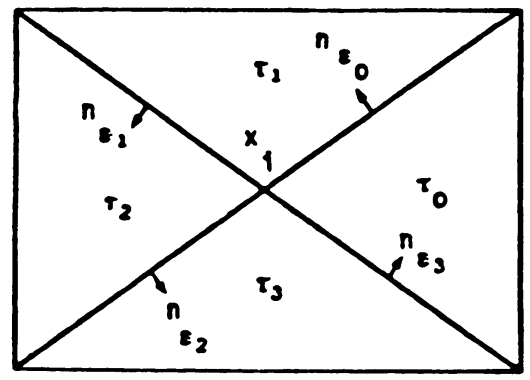

Figure 4.1 


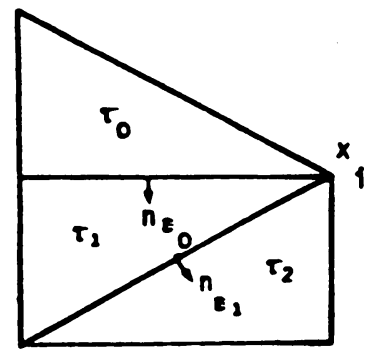

Figure 4.2

Inequality (4.6) holds in like manner for all the other coefficients, including the ones associated with boundary vertices. For example, for the boundary vertex $x_{i}$ depicted in Figure 4.2, the resulting system of linear equations is

$$
\left[\begin{array}{rr}
1 & 0 \\
-1 & 1 \\
0 & -1
\end{array}\right]\left[\begin{array}{l}
\theta_{\varepsilon_{0}, j_{0}} \\
\theta_{\varepsilon_{1}, j_{1}}
\end{array}\right]=\left[\begin{array}{c}
-F_{\tau_{0}}\left(b_{i}\right) \\
-F_{\tau_{1}}\left(b_{i}\right) \\
-F_{\tau_{2}}\left(b_{i}\right)
\end{array}\right] .
$$

Again, the system is consistent because $F\left(b_{i}\right)=0$, and this time the unique solution is

$$
\boldsymbol{\theta}_{\varepsilon_{m}, j_{m}}=-\sum_{k=0}^{m} F_{\tau_{k}}\left(b_{i}\right) .
$$

With these choices for the coefficients of $\theta$, we have

THEOREM 4.2. If $u_{H}$ is in $H^{2}(\tau)$ for all $\tau$,

$$
\left|h_{\varepsilon}^{1 / 2} \theta\right|_{E} \leqslant C_{8}\left\|\left|e_{H}\right|\right\|_{\tau},
$$

where $C_{8}=C_{8}\left(\bar{a}, \bar{b}, \delta_{0}, C_{3}\right)$.

Proof. On any edge $\varepsilon$, since $\left|\sigma_{\varepsilon, j}\right|_{\varepsilon}=2 h_{\varepsilon}^{-1 / 2}$, by the definition of $\theta$ and (4.6),

$$
\left|h_{\varepsilon}^{1 / 2} \theta\right|_{E_{\tau}} \leqslant 4 \sum_{x_{i} \in \bar{\tau}} \sum_{\tau^{\prime} \in \operatorname{supp}\left(b_{i}\right)}\left|F_{\tau^{\prime}}\left(b_{i}\right)\right| .
$$

By (3.2) and (3.7), since

$$
\begin{gathered}
a \frac{\partial e_{H}}{\partial n_{\tau}}=\left[a \frac{\partial e_{H}}{\partial n_{\tau}}\right]_{A}+\frac{1}{2}\left[a \frac{\partial U}{\partial n_{\tau}}\right]_{J}, \\
F_{\tau}\left(b_{i}\right)=a\left(e_{H}, b_{i}\right)_{\tau}-\left\langle\left[a \frac{\partial e_{H}}{\partial n_{\tau}}\right]_{A}, b_{i}\right\rangle_{E_{\tau} \cap E_{I}} .
\end{gathered}
$$

Since

$$
\left|b_{i}\right|_{\varepsilon}=\left(h_{\varepsilon} / 3\right)^{1 / 2} \text { and }\|\| b_{i} \|_{\tau} \leqslant C_{6} \equiv\left(2 \bar{a} / \delta_{0}^{2}+\bar{b} h^{2}\right)^{1 / 2} \text {, }
$$

then

$$
\left|\left(F_{\tau}\left(b_{i}\right)\right)\right| \leqslant C_{6}\left\|\left.e_{H}\left|\|_{\tau}+3^{-1 / 2}\right| h_{\varepsilon}^{1 / 2}\left[a \frac{\partial \varepsilon_{H}}{\partial n_{\tau}}\right]_{A}\right|_{E_{\tau} \cap E_{l}} .\right.
$$


By (4.7) and Lemma 3.1,

$$
\begin{aligned}
\left|h_{\varepsilon}^{1 / 2} \theta\right|_{E_{I}}^{2} & =\frac{1}{2} \sum_{\tau \in T}\left|h_{\varepsilon}^{1 / 2} \theta\right|_{E_{\tau} \cap E_{I}}^{2} \leqslant \frac{1}{2}\left(12 C_{7}\right)^{2} \sum_{\tau \in T} \sup _{x_{i} \in \tau}\left|F_{\tau}\left(b_{i}\right)\right|^{2} \\
& \leqslant\left(12 C_{7}\right)^{2}\left\{C_{6}^{2} \| e_{H}||^{2}+\frac{1}{3}\left|h_{\varepsilon}^{1 / 2}\left[a \frac{\partial e_{H}}{\partial n}\right]_{A}\right|_{E_{I}}^{2}\right\} \\
& \leqslant\left(12 C_{7}\right)^{2}\left(C_{6}^{2}+C_{3}^{2} / 3\right)\left\|e_{H}\right\|^{2},
\end{aligned}
$$

where $C_{7}=C_{7}\left(\delta_{1}\right)$ is the maximum number of triangles sharing a vertex.

We are now ready to bound $\|\hat{e}\| \|$.

THEOREM 4.3. If $u_{H}$ is in $H^{2}(\tau)$ for all $\tau$ in $T$, and $\theta$ is chosen such that (4.5) and Theorem 4.2 hold, then

$$
\|\hat{e}\| \leqslant\left(1+C_{\hat{e}}\right)\left\|e_{H}\right\|,
$$

where $C_{\hat{e}}=\left(C_{3}+C_{8}\right)\left|h_{\varepsilon}^{-1 / 2}[\hat{e}]_{J}\right|_{E_{l}} /\|\hat{e}\| \| \leqslant C_{9}$.

Proof. By (3.8) and (4.2), we have

$$
a(\hat{e}, v)=a\left(e_{H}, v\right)+\left\langle\theta-\left[a \frac{\partial e_{H}}{\partial n}\right]_{A},[v]_{J}\right\rangle_{E_{I}}
$$

for all $v$ in $S_{T}$. Taking $v=\hat{e}$, we obtain, using Lemma 3.1 and Theorem 4.2,

$$
\|\hat{e}\|\left\|^{2} \leqslant\right\| e_{H}\|\|\|\hat{e}\|+C_{\hat{e}}\left\|e_{H}\right\|\|\| \hat{e} \| .
$$

To see that $C_{\hat{e}}$ is bounded, note that by (4.5), in each element $\tau, a(\hat{e}, v)_{\tau}=0$ for any linear polynomial $v$. A standard duality argument then implies

Thus, by Lemma 3.2,

$$
\|\hat{e}\|_{\tau} \leqslant C_{4}\left(a, b, \delta_{1}\right) h_{\tau}\|\hat{e}\|_{\tau} .
$$

$$
C_{\hat{e}} \leqslant\left(C_{3}+C_{8}\right) C_{5} \equiv C_{9} .
$$

The term $C_{\hat{e}}$ measures the discontinuity of $\hat{e}$; in particular, if $\hat{e}$ is continuous, $C_{\hat{e}}=0$ (i.e., $\hat{e}=e_{\bar{S}}$ ). We could force $\hat{e}$ to converge to $e_{\bar{S}}$ by penalizing its jumps in value across the interelement boundaries $E_{I}$, but the penalties would necessarily destroy the local nature of the problems (4.1), and thus make the computation of $\hat{e}$ much more expensive.

5. A Second Error Estimator. One of the prime considerations in choosing the function $\theta$ in Section 4 was to make the local problems (4.1) consistent. In this section, we present an alternative algorithm for computing error indicators $\tilde{e}$ in $\bar{S}_{T}$ which avoids the computation of $\theta$. The function $\tilde{e}$ is defined on $\tau$ in $T$ as the solution of the local problem

$$
a(\tilde{e}, v)_{\tau}=F_{\tau}(v-I v) \text { for all } v \text { in } \bar{S}_{\tau} .
$$

Since $1=I(1)$, Eqs. (5.1) are consistent. If $b \equiv 0$ on $\tau$, we require

$$
\iint_{\tau} \tilde{e} d x=0
$$

to insure a unique solution. Summing over $\tau$, we obtain the global definition of $\tilde{e}$ in $\bar{S}_{T}$ :

$$
a(\tilde{e}, v)=F(v-I v) \text { for all } v \text { in } \bar{S}_{T} .
$$

THEOREM 5.1. $\left(1-\beta^{2}\right)^{1 / 2}\left\|\left|e_{H}\|\| \leqslant\|\tilde{e}\|\right|+C_{0}\right\| e_{S} \|$. 
Proof. From (5.2) and (3.4) with $Q=\bar{S}, v=e_{\bar{S}}$,

$$
a\left(\tilde{e}, e_{\bar{S}}\right)=a\left(e_{\bar{S}}, e_{\bar{S}}-I e_{\bar{S}}\right) .
$$

Using (3.5b) and the fact that $I e_{\bar{S}}$ is in $\underline{S} \subseteq S$,

$$
\begin{aligned}
\left\|e_{\bar{S}}\right\|^{2} & =a\left(e_{\bar{S}}, e_{\bar{S}}\right)=a\left(\tilde{e}, e_{\bar{S}}\right)+a\left(e_{\bar{S}}, I e_{\bar{S}}\right) \\
& \leqslant\|\tilde{e}\|\left\|e_{\bar{S}}\right\|+C_{0}\left\|e_{S}\right\|\left\|e_{\bar{S}}\right\| .
\end{aligned}
$$

Theorem 5.1 follows by dividing by $\left\|\left|e_{\bar{S}}\right|\right\|$ and using Lemma 3.3.

THEOREM 5.2. If $u_{H}$ is in $H^{2}(\tau)$ for all $\tau$ in $T$,

$$
\|\tilde{e}\| \leqslant\left(1+C_{\tilde{e}}\right)\left\|e_{H}\right\|\left\|+C_{0}\right\| e_{S} \|,
$$

where

$$
C_{\tilde{e}}=\left[C_{0}+C_{3} C_{5}\left(1+C_{0}\right)\right] \inf _{\chi \in \bar{S}} \frac{\|\tilde{e}-\chi\| \|}{\|\tilde{e}\|} .
$$

Proof. From (3.8) and (5.2),

$$
a(\tilde{e}, v)=a\left(e_{H}, v-I v\right)-\left\langle\left[a \frac{\partial e_{H}}{\partial n}\right]_{A},[v-I v]_{J}\right\rangle_{E_{I}}
$$

for all $v$ in $\bar{S}_{T}$. Take $v=\tilde{e}-\phi$ where $\phi$ is the elliptic projection of $\tilde{e}$ onto $\bar{S}$, so that

$$
\|\tilde{e}-\phi\|=\inf _{\chi \in \bar{S}}\|\tilde{e}-\chi\| \text { and }\|\phi\| \leqslant\|\tilde{e}\| .
$$

Noting that $a(\tilde{e}, \phi)=a\left(e_{H}, \phi-I \phi\right)$, we obtain

$$
\begin{aligned}
a(\tilde{e}, \tilde{e})= & a\left(e_{H}, \tilde{e}\right)-a\left(e_{H}, I(\tilde{e}-\phi)\right)-a\left(e_{H}, I \phi\right) \\
& -\left\langle\left[a \frac{\partial e_{H}}{\partial n}\right]_{A},[\tilde{e}-\phi-I(\bar{e}-\phi)]_{J}\right\rangle_{E_{I}} .
\end{aligned}
$$

We now bound the four terms on the right-hand side of (5.4). The first two are trivial:

$$
\begin{gathered}
\left|a\left(e_{H}, \tilde{e}\right)\right| \leqslant\left\|e_{H}\right\| \mid\|\tilde{e}\| ; \\
\left|a\left(e_{H}, I(\tilde{e}-\phi)\right)\right| \leqslant C_{0}\left\|\left|e_{H}\right|\right\| \mid\|\tilde{e}-\phi\| \| .
\end{gathered}
$$

For the third, note $I \phi$ is in $\underline{S}$. Using (3.5a),

$$
\left|a\left(e_{H}, I \phi\right)\right|=\left|a\left(e_{S}, I \phi\right)\right| \leqslant C_{0}\left\|e_{S}\right\|\|\| \tilde{e} \| .
$$

The fourth term uses Lemmas 3.1-3.2. Note that $\tilde{e}-\phi-I(\tilde{e}-\phi)$ is zero at the interpolation points, so that the hypothesis of Lemma 3.2 is satisfied. Thus, we obtain

$$
\left|\left\langle\left[a \frac{\partial e_{H}}{\partial n}\right]_{A},[\tilde{e}-\phi-I(\tilde{e}-\phi)]_{J}\right\rangle_{E_{l}}\right| \leqslant C_{3} C_{5}\left(1+C_{0}\right)\left\|\mid e_{H}\right\|\|\| \tilde{e}-\phi\|\| .
$$

Theorem 5.2 follows from (5.4)-(5.8).

If $e_{S}$ satisfies (2.6), the bounds are somewhat stronger.

THEOREM 5.3. If (2.6) is satisfied and $u_{H}$ is in $H^{2}(\tau)$ for all $\tau$ in $T$, then

$$
\left(1-\beta^{2}\right)^{1 / 2}\left\|e_{H}\right\| \leqslant\|\tilde{e}\| \leqslant\left(1+C_{\tilde{e}}\right)\left\|e_{H}\right\| .
$$

Proof. In this case, the last term in (5.3) and the term bounded in (5.7) are both zero. 
In analogy with Theorem $4.3, C_{\tilde{e}}$ measures the discontinuity of $\tilde{e}$; if $\tilde{e}$ is continuous, $C_{\tilde{e}}=0$. Except for the global use of $(2.5)$, we could have proved a local analogue of Theorem 5.2 for each element.

6. A Third Error Estimator. Equations (5.1) define the error indicator $\tilde{e}$ on $\tau$ as the solution of a linear system with size equal to $\operatorname{dim}\left(\bar{S}_{\tau}\right)$, the dimension of $\bar{S}_{\tau}$. In this section, we present an alternative algorithm for computing an error indicator $\check{e}$ on $\tau$ which is the solution of a smaller linear system which is automatically positive-definite.

The error indicator $\check{e}$ in $\check{S}_{\tau}$ is defined on $\tau$ in $T$ as the solution of the local problem

$$
a(\check{e}, v)_{\tau}=F_{\tau}(v) \text { for all } v \text { in } \check{S}_{\tau} \text {. }
$$

Since the constant function 1 is not in $\check{S}_{\tau}$, the linear system (6.1) is positive-definite. Note that $\operatorname{dim}\left(\check{S}_{\tau}\right)=\operatorname{dim}\left(\bar{S}_{\tau}\right)-\operatorname{dim}\left(\underline{S}_{\tau}\right)$. By the definition of $\check{S}_{\tau}$ and (5.1),

$$
a(\check{e}, v)=F_{\tau}(v-I v)=a(\tilde{e}, v) \text { for all } v \text { in } \check{S}_{\tau} \text {. }
$$

Equation (6.2) shows that $\check{e}$ is the elliptic projection of $\tilde{e}$ into $\check{S}_{\tau}$.

THEOREM 6.1. $(1-\gamma)^{1 / 2}\|\tilde{e}\||\leqslant\|\check{e}\||$.

Proof. Let $\tilde{e}=\tilde{e}_{1}+\tilde{e}_{2}$, where $\tilde{e}_{1}$ is in $\underline{S}_{T}$ and $\tilde{e}_{2}$ is in $\check{S}_{T}$. By Lemma 3.4,

$$
\|\tilde{e}\|^{2}=a\left(\tilde{e}_{1}, \tilde{e}_{1}\right)+2 a\left(\tilde{e}_{1}, \tilde{e}_{2}\right)+a\left(\tilde{e}_{2}, \tilde{e}_{2}\right) \geqslant(1-\gamma)\left\|\tilde{e}_{2}\right\|^{2} .
$$

Since $\tilde{e}_{1}=I \tilde{e}_{1}, a\left(\tilde{e}, \tilde{e}_{1}\right)=0$. Then by (6.2), with $v=\tilde{e}_{2}$,

$$
\|\tilde{e}\|^{2}=a\left(\tilde{e}, \tilde{e}_{1}\right)+a\left(\tilde{e}, \tilde{e}_{2}\right)=a\left(\check{e}^{2}, \tilde{e}_{2}\right) \leqslant\|\check{e}\|\|\| \tilde{e}_{2}\|\|,
$$

and the theorem follows.

By (6.2) with $v=\check{e}$, we immediately have

THEOREM 6.2. $\|\check{e}\||\leqslant|\|\tilde{e}\|$.

Using Theorems 5.1-5.3 and 6.1-6.2, we obtain

TheOREM 6.3. $\left(1-\beta^{2}\right)^{1 / 2}\left\|\left|e_{H}\left\|\left|\leqslant(1-\gamma)^{-1 / 2}\|\check{e}\|\right|+C_{0}\right\| e_{S} \|\right.\right.$.

If $u_{H}$ is in $H^{2}(\tau)$ for all $\tau$ in $T$,

$$
\|\check{e}\|\left\|\leqslant\left(1+C_{\tilde{e}}\right)\right\| e_{H}\|\|+C_{0}\left\|e_{S}\right\| .
$$

If, in addition, (2.6) is satisfied, then

$$
\left\{\left(1-\beta^{2}\right)(1-\gamma)\right\}^{1 / 2}\left\|e_{H}\right\| \leqslant\|\ddot{e}\|\left\|\leqslant\left(1-C_{\tilde{e}}\right)\right\| e_{H} \| .
$$

7. Numerical Results. In this section, we present some example calculations comparing the error estimators $\|\hat{e}\|\|,\| \tilde{e} \|$, and $\|\check{e}\|$, described in Sections 4, 5, and 6, respectively. In these calculations, $\underline{S}=S$ is the space of $C^{0}$ piecewise-linear triangular finite elements, and $\bar{S}$ is the space of $C^{0}$ piecewise-quadratic elements. The test problems are of the form

$$
\begin{array}{rlrl}
-\Delta u & =0 & r l & =0 \\
\frac{\partial u}{\partial n} & =\frac{2}{k} \cos \left(\frac{2 \theta}{k}\right) & & \text { on } \partial \Omega_{2}, \\
\frac{\partial u}{\partial n} & =0 & & \text { in } \Omega, \\
& & & \text { on } \partial \Omega_{3},
\end{array}
$$


where $\Omega=\{(r, \phi): 0<r<1,0<\theta<k \pi / 4\} ; \partial \Omega_{1}$ is the line $\theta=0,0 \leqslant r \leqslant 1 ; \partial \Omega_{2}$ is the arc $0 \leqslant \theta \leqslant k \pi / 4, r=1 ; \partial \Omega_{3}$ is the line $\theta=k \pi / 4,0 \leqslant r \leqslant 1 ;(r, \theta)$ are polar coordinates; and $k=1,3,4$, and 8 . The solution of (7.1) is

$$
u_{H}=r^{2 / k} \sin \left(\frac{2 \theta}{k}\right)
$$

the leading term of the point singularity for a corner with interior angle $k \pi / 4$, so that $u_{H}$ is in $H^{1+2 / k-\varepsilon}(\Omega)$ for any $\varepsilon>0$, but not for $\varepsilon=0$, when $k=3$, 4 , or 8 [15]. When $k=3,4$, or 8 , the analogues to Theorems $4.2,4.3,5.2,5.3$, and 6.3 must use weaker bounds on $\|\mid \bar{e}\|_{\tau}$ in element touching the origin, and count on adaptive mesh refinement to make those elements very small.

The problems were solved using the Fortran package PLTMG [11]. This code used local adaptive mesh refinement and created for each problem a sequence of meshes of varying degrees of nonuniformity. The meshes for the case $k=8$ are illustrated in Figure 7.1. PLTMG uses a multi-level iterative method for solving the linear systems and typically generates approximate solutions $U$ in $S$ such that $\left\|\left|e_{S}\|\|=\left\|U-u_{S}\right\|\right|\right.$ is somewhat less than the discretization error.

For each problem and for each mesh, we computed the quantities $\left\|e_{H}\right\|,\|\hat{e}\| \|$, $\|\tilde{e}\| \mid$, and $\|\check{e}\| \mid$. For $\left\|\left|e_{H} \|\right|\right.$, a numerical quadrature rule using 6 quadrature points per element was used; $\hat{e}, \tilde{e}$, and $\check{e}$ were all piecewise-quadratic polynomials and their norms were computed exactly, except for small errors incurred for elements with curved boundary edges.

The effectivity index [9] or efficiency index [10] for an error estimator $\|\bar{e}\|, \bar{e}=\hat{e}$, $\tilde{e}$, or $\check{e}$, is defined as

$$
\operatorname{eff}(\bar{e})=\|\bar{e}\| /\left\|e_{H}\right\| .
$$
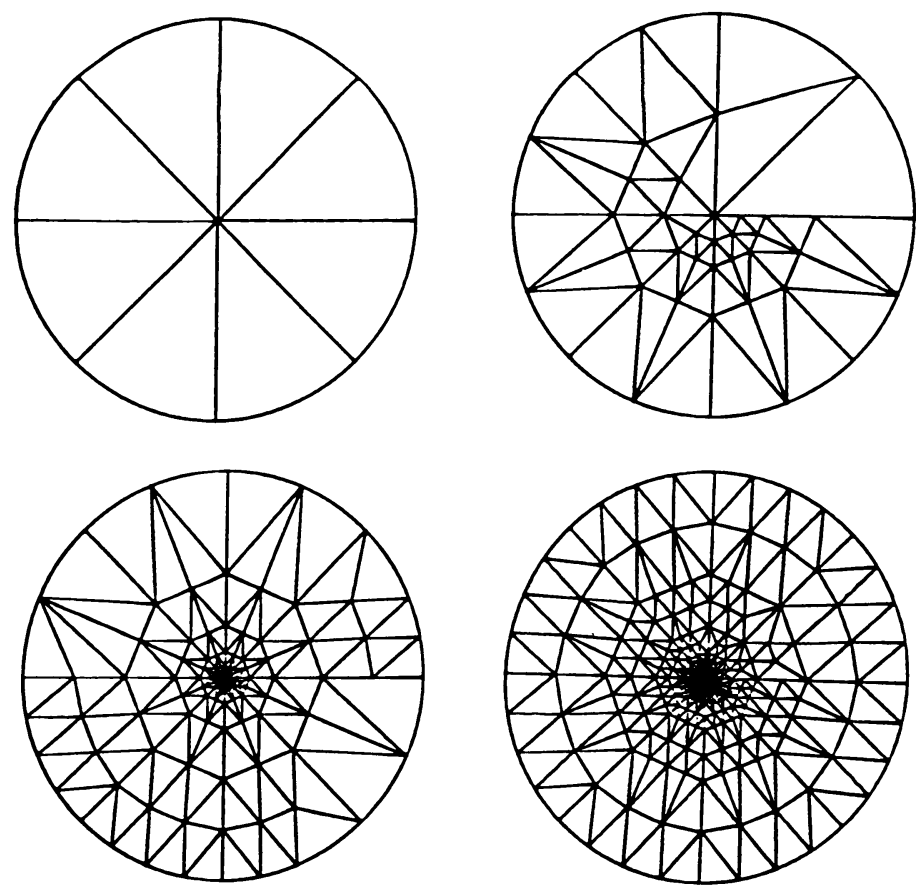

FIGURE 7.1 
We chose to measure the relative error

$$
\rho(\bar{e})=\operatorname{eff}(\bar{e})-1 .
$$

Note that if $\bar{e}$ satisfies (1.1), we have $-\varepsilon_{1} \leqslant \rho(\bar{e}) \leqslant \varepsilon_{2}$.

Having $\rho(\bar{e})$ near or converging to zero is clearly the most desirable situation. Positive values of $\rho$ indicate an overestimate of the true error and are acceptable as long as $\rho$ is not too much larger than one. Negative values of $\rho$ mean the error indicator has given an erroneously optimistic estimate of $\left\|e_{H} \mid\right\|$. In Table 7.1 we tabulate the values of $\rho(\bar{e})$ for each error indicator on each mesh for each test case. $\mathrm{NV}$ is the number of vertices in the mesh.

On the basis of Theorems 4.1, 5.1 and 6.3, we expect $\rho(\hat{e}) \geqslant 0, \rho(\tilde{e}) \geqslant 0$, and $\rho(\check{e}) \geqslant(1-\gamma)^{1 / 2}-1$, since once the initial mesh is appreciably refined, the effects of $\beta$ and $\left\|e_{S}\right\| \mid$ are small. For all error indicators we have $\rho(\bar{e}) \leqslant \varepsilon_{2}$, where the data suggest $\varepsilon_{2} \leqslant 3$ for this problem class.

The apparent convergence of $\rho(\check{e})$ to zero represents a particularly nice state of affairs, not only because it means $\|\check{e}\| \|$ is quite accurate, but also because $\check{e}$ is the least costly of the three indicators to compute.

TABLE 7.1

$\begin{array}{rrlcll}k & N V & \left\|e_{H}\right\| & \rho(\hat{e}) & \rho(\tilde{e}) & \rho(\check{e}) \\ 1 & 3 & .428 & .247 & .150 & .121 \\ & 15 & .129 & .508 & .385 & .0117 \\ & 45 & .0653 & .681 & .430 & .00366 \\ & 153 & .0328 & .974 & .452 & .00131 \\ & 561 & .0164 & 1.44 & .463 & .000480 \\ 3 & 5 & .241 & .408 & .152 & -.0960 \\ & 17 & .123 & .520 & .335 & .0591 \\ & 75 & .0522 & .998 & .625 & .196 \\ & 137 & .0379 & 1.24 & .697 & .255 \\ & 480 & .0200 & 1.50 & .589 & .154 \\ & 1908 & .00946 & 2.23 & .579 & .110 \\ 4 & 6 & .368 & .618 & .0166 & -.199 \\ & 27 & .181 & .579 & .335 & .0280 \\ & 119 & .0889 & .695 & .522 & .135 \\ & 501 & .0389 & .842 & .588 & .148 \\ & 1363 & .0225 & .959 & .541 & .0963 \\ & 13 & .499 & 1.46 & -.230 & -.390 \\ 8 & 10 & .368 & 1.87 & .0545 & -.197 \\ & 41 & .208 & 1.22 & .407 & .0645 \\ & 161 & .120 & 1.32 & .378 & .0263\end{array}$

The calculation of $\hat{e}$ is the most expensive since it involves solving a linear system like (4.5) for each vertex in order to obtain $\theta$. We must then assemble and solve a symmetric positive-semidefinite $6 \times 6$ linear system (of rank 5) in each element. The computation of $\tilde{e}$ also involves assembling and solving a $6 \times 6$ linear system in each 
element, but the calculation of $\theta$ is avoided. For $\check{e}$ only a $3 \times 3$ symmetric positive-definite linear system (corresponding to the three edge midpoint Lagrange basis functions) is assembled and solved.

From other tests using square elements [16], we have observed apparent convergence of $\rho(\hat{e})$ and $\rho(\tilde{e})$ to zero for some test problems. Under suitable assumptions, Babuška and Miller [4] have recently proven convergence of the error estimators used in their two-dimensional code (with piecewise-bilinear basis functions) to the norm of the true error.

For problems in one space dimension, when $S$ consists of piecewise-linear functions, the formulations leading to $\hat{e}$ and $\tilde{e}$ are equivalent. When, in addition, $\bar{S}$ consists of piecewise-quadratic functions, the analogue of $\check{e}$ is only slightly different from an error indicator of Babuška and Rheinboldt ( $\|\check{e}\|_{\tau}$ is analogous to $\hat{\eta}_{j}(\Delta)$ in [10]). When, in addition, $a(x)=1$ and $b(x)=0$, all three indicators are equivalent. Under suitable assumptions, it can be shown that the corresponding one-dimensional error estimators converge to the norm of the true error.

Acknowledgments. This work was initiated while the first author was a summer professor at Exxon Production Research Company. We are grateful to the management of EPR for permission to publish this paper, and to Linda Scott and Marie Mason of EPR for help in preparation of the manuscript.

This research was supported in part by the Office of Naval Research through contracts N00014-82-K-0197 (University of California at San Diego) and N0001476-C-0277 (Yale University).

Department of Mathematics

University of California, San Diego

La Jolla, California 92093

Exxon Production Research Company

Houston, Texas 77001

1. S. Agmon, Lectures on Elliptic Boundary Value Problems, Van Nostrand, New York, 1965.

2. I. BABUSKKa, Private Communication.

3. I. BABUSKA \& M. LUSKIN, "An adaptive time discretization procedure for parabolic problems," in Proc. Fourth IMACS Internat. Sympos. on Computer Methods for Partial Differential Equations, Lehigh University, Bethlehem, Pennsylvania, 1981, pp. 5-8.

4. I. BabuSKa \& A. Miller, A Posteriori Error Estimates and Adaptive Techniques for the Finite Element Method, Technical Report BN-968, Institute for Physical Science and Technology, University of Maryland, 1981.

5. I. BABUSKA \& W. C. RheinboldT, "A posteriori error analysis of finite element solutions for one-dimensional problems,” SIAM J. Numer. Anal., v. 18, 1981, pp. 565-589.

6. I. BABUSKa \& W. C. Rheinboldt, "A posteriori error estimates for the finite element method," Internat. J. Numer. Methods Engrg., v. 12, 1978, pp. 1597-1615.

7. I. BabuSKa \& W. C. Rheinboldt, “Analysis of optimal finite-element meshes in $R^{1}$," Math. Comp., v. 33, 1979, pp. 435-463.

8. I. BABUSKA \& W. C. RheinBoldT, "Error estimates for adaptive finite element computations," SIAM J. Numer. Anal., v. 15, 1978, 736-754.

9. I. BABUSKa \& W. C. Rheinboldt, "On the reliability and optimality of the finite element method," Comput. \& Structures, v. 10, 1979, 87-94.

10. I. BABUSKA \& W. C. RHEINBOLDT, "Reliable error estimation and mesh adaptation for the finite element method," in Computational Methods in Nonlinear Mechanics, North-Holland, New York, 1980, pp. 67-108.

11. R. E. BANK, PLTMG Users' Guide, June, 1981 version, Technical Report, Department of Mathematics, University of California, San Diego, 1982. 
12. R. E. Bank \& T. F. Dupont, Analysis of a Two-Level Scheme for Solving Finite Element Equations, Technical Report, University of Texas Center for Numerical Analysis, 1980.

13. R. E. Bank \& A. H. Sherman, "A multi-level iterative method for solving finite element equations," in Proc. Fifth Symposium on Reservoir Simulation, Society of Petroleum Engineers of AIME, Dallas, 1979, pp. 117-126.

14. P. Percell \& M. F. Wheeler, "A local residual finite element procedure for elliptic equations," SIAM J. Numer. Anal., v. 15; 1978, pp. 705-714.

15. G. Strang \& G. J. Fix, An Analysis of the Finite Element Method, Prentice-Hall, Englewood Cliffs, N. J., 1973.

16. A. WeISER, Local-Mesh, Local-Order, Adaptive Finite Element Methods With A Posteriori Error Estimators for Elliptic Partial Differential Equations, Technical Report 213, Computer Science Department, Yale University, 1981. 